\title{
NY-ESO-1: a promising cancer testis antigen for sarcoma immunotherapy and diagnosis
}

\author{
Stephen M. Smith, O. Hans Iwenofu \\ Department of Pathology \& Laboratory Medicine, The Ohio State University Wexner Medical Center, Columbus, OH, USA \\ Contributions: (I) Conception and design: OH Iwenofu; (II) Administrative support: OH Iwenofu; (III) Provision of study materials or patients: None; \\ (IV) Collection and assembly of data: SM Smith; (V) Data analysis and interpretation: All authors; (VI) Manuscript writing: All authors; (VII) Final \\ approval of manuscript: All authors. \\ Correspondence to: O. Hans Iwenofu, MD. Department of Pathology \& Laboratory Medicine, Wexner Medical Center at The Ohio State University, \\ 410 West 10th Avenue, Columbus, OH 43210, USA. Email: Hans.Iwenofu@osumc.edu.
}

\begin{abstract}
Sarcomas are heterogenous malignant mesenchymal neoplasms. These are often notoriously difficult to treat particularly in the metastatic setting. There is therefore an urgent need for development of better and more efficacious targeted therapies. Cancer testis antigens (CTAs) are a family of proteins in which aberrant gene-activation and subsequent high level mRNA expression, are restricted to testicular germ cells and are seen in certain malignancies. Importantly, the restriction of this class of antigens to testicular germ cells and malignancies and not somatic tissue, makes them an excellent choice for targeted immunotherapy. The NY-ESO-1 is the most immunogenic of CTA and has, of late, become well-studied for its diagnosis and potential treatment implications in sarcomas. This paper reviews both the role of NYESO-1 in the diagnosis of sarcomas, as well as the implications of this CTA in vaccine development and treatment of sarcomas.
\end{abstract}

Keywords: NY-ESO-1; sarcoma

Submitted Jul 28, 2018. Accepted for publication Aug 23, 2018.

doi: $10.21037 /$ cco.2018.08.11

View this article at: http://dx.doi.org/10.21037/cco.2018.08.11

\section{Introduction}

The 1908 Nobel Prize in Medicine and Physiology was, in part, awarded to a German immunologist names Paul Ehrlich for his "work on immunity". Within the next year, he would develop an idea: could carcinogenesis be specifically targeted utilizing an immunologic approach (1). The idea drifted in and out of vogue until the 1950s when tumor-specific immunity peaked interest again (2). From then, vast knowledge has been gained in the immunologic basis of neoplasia, including not only mechanisms by which the immune system combats cancer cells, but also how those same cancer cells may morph to evade the same system. Recent advances in the past several decades have led to immunologic targeting of cancer, the basis of this discussion.

Although external antigens could be readily identified by autologous and allogeneic serologic methods, until the late 1990 's, the serum titers were generally too low to permit the molecular identification of the detected antigens. The use of serological identification of antigens by recombinant expression cloning (SEREX) by Chen et al. in 1997 lead to the discovery of intracellular antigens in a set of esophageal tumors (3). Among these were primitive antigens aberrantly expressed after the cell's return to a primordial state, including the NY-ESO-1 protein.

\section{The role of NY-ESO-1 cancer testis antigen (CTA) in development}

CTAs are a family of proteins which share three common characteristics: (I) predominant normal expression of the pre-cursor mRNA is found in testicular tissue and typically not in other normal tissues; (II) aberrant gene-activation 
and subsequent high level mRNA expression in certain malignancies; and (III) lineage-nonspecific expression when arising in a malignant setting (4).

Given the innate immunologic barriers within the testis which prevent immune response to genetically "foreign" material, re-expression of a primitive embryologic protein by tumor cells generates the potential for $\mathrm{T}$-cell recognition of these antigens. Early genes identified included the $M A G E$ and $G A G E$ families of proteins, first found due to their expression within tumoral cells which were recognized by cytotoxic T-cells (5-7).

The implication of NY-ESO-1 as a tumor antigen was first described by Chen et al. in 1997, using prokaryotictransfected cDNA from an esophageal squamous cell carcinoma patient as an antigenic target within that patient's serum. The humoral response was measured and the corresponding antibodies identified, identifying tumoral targets which generated a clear cell-mediated response. After identifying NY-ESO-1, humoral response to this antigen within various tumors was sought; many melanomas, breast carcinomas and bladder carcinomas (among others) showed a positive response. Of note, two leiomyosarcomas and two other sarcomas were negative for NY-ESO-1-induced humoral response in that study (4).

Jungbluth et al. first documented normal expression of the NY-ESO-1 antigen in normal human testis (8). NY-ESO-1 can be identified at 18 weeks gestation in testicular gonocytes, similar to MAGE-A4 CTA (9). Within developing spermatogonia, $N Y-E S O-1$ was identified as one of nine genes upregulated during differentiation, suggesting a role in spermatogonial clonal expansion (10). Little is known, however, about the physiology of NYESO-1 within spermatogonia. Expression beyond meiosis I of spermatogenesis has not been shown; this CTA is also not expressed in Sertoli cells (9). Chen et al. have shown the NY-ESO-1 protein to arise from a gene region on chromosome $\mathrm{Xq} 28$, within the region where other CTA (MAGE family members) are encoded (3).

\section{CTA, NY-ESO-1 and cancer pathobiology}

The NY-ESO-1 gene on chromosome Xp28 has approximately $679 \mathrm{bp}(3)$ and encodes a $22 \mathrm{kDa}$ protein derived from three exons within the gene. Of the protein domains studied, perhaps the most intriguing was derived from the S. cerevisiae homologue, Pcc1p (11). Alpen et al. provided data supporting the evolutionary conservation of the NY-ESO-1 gene family [though later work by Dobrynin et al. found that most CTA are novel or primitive (12)] suggesting significance in cell survival and/or replication (13). Working from that data, Kisseleva-Romanova et al. sought to identify the necessity of the Pcclp protein, showing that this NY-ESO-1 homologue functions as a transcription factor for several major proteins needed during cell cycle regulation and produces cell death when absent (11). Within mammalian cells, other avenues have been pursued to identify the pathobiology of NY-ESO-1. In melanoma cell lines, Cho et al. demonstrated that the interactions of NY-ESO-1 with a members of the melanoma antigen family (MAGE family), MAGE-C1, which is another protein family expressed during spermatogenesis (14-16). Co-localization of both proteins was observed via indirect immunofluorescence studies in the same cell population. However, while melanoma cells appear to express NY-ESO-1 in both the nucleus and cytoplasm of the cell; normal mesenchymal stem cells (MSCs) appear to show exclusive nuclear NY-ESO-1 immunoreactivity (17). Although the direct physiologic functions of MAGE are equally as poorly-understood as those of NY-ESO-1, this association is significant as MAGE proteins have documented cell division activity with therapeutically targetable stages. In non-small cell lung carcinoma, breast carcinoma and hepatocellular carcinoma cell lines, Cappell et al. showed that the MAGE family was among a cohort of gametogenic proteins required for chromosome segregation and microtubule formation during tumor formation and development (18). Other CTA have been implicated in microtubule formation and development, with increased expression being associated with microtubule-inhibitor antineoplastic therapy resistance (e.g., paclitaxel) (19).

\section{Cellular and humoral T-cell responses to NY- ESO-1}

NY-ESO-1 became of prime interest after it was determined that this protein represented a prime epitope and, therein, a target for vaccine development. As noted previously, the immunogenic barrier of the testis should, theoretically prevent the presence of anti-NY-ESO-1 (a-NE1) antibody development in a normal human individual. Studies in the general population identified a frequency of anti-NY-ESO-1 T-cells as having a frequency between 0.5 and 5 precursors per one million CD4+ T-cells, with the vast majority of these T-cells exhibiting reactivity to the previously described "hotspots" of overlapping sequences exhibiting higher binding capacity for multiple frequently expressed MHC class II alleles $(20,21)$. Liu et al. demonstrated in mice that NY- 
ESO-1 binding to immature dendritic cells was dependent on its polymeric structure, requiring Toll-like receptor-4 (TLR4) activation (22). Analysis of a melanoma patient exhibiting high titers of a-NE1 revealed that simultaneous cellular and humoral responses were occurring within the same patient (23). The strong binding interaction of the polymeric structure with TLR4 promotes $\mathrm{T}_{\mathrm{H}}$-cell interactions with B-cells promote a-NE1 production (22). And, as expected, a strong cytotoxic T-cell response is elicited from the activated humoral immunity system-so much so that enzyme-linked immunospot (ELISPOT) assays show strong concordance and are useful in monitoring NY-ESO-1 vaccine efficacy (24).

Analysis of amino acid sequences within NY-ESO-1 has further characterized these highly antigenic regions. Neumann et al. identified a pentadecameric region (p134148) which stimulated $T$-cell responses restricted to the HLA-DRB1 subtypes *0101, *0301, ${ }^{*} 0401$, and ${ }^{*} 0701$ (25). This sequence induced responses in four of 38 cancer patients tested, apparently independent of serum a-NE1 titers. The p157-165 peptide sequence was found to be efficaciously recognized by cytotoxic T-cells in melanoma patients (26). The same study also showed that p157-167 and p155-163 peptides were less efficient, despite increased binding affinity to HLA-A*201. Other studies have shown that the p157-167 is likely the dominant peptide recognized by T-cells, targeting HLA-A2 T-cells specifically (23). Fonteneau and colleagues sought to determine the method by which the NE1 p157-167 highly antigenic sequence was processed by antigen-presenting cells for interactions with T-cells (27). Interestingly, in lieu of more common proteosome or autophagy-dependent endogenous processing pathways, a combination of intercellular antigen transfer amongst tumor cells and macroautophagy was found to be utilized in this interaction.

Sommermeyer et al. identified three types of T-cells with in vitro-designed $\mathrm{T}$-cell receptors exhibiting a-NE1 potential; the most promising of which displayed moderate efficiency in antigen binding but a precise peptidespecific performance in the functional assays assessed (28). Capitalizing on these studies, Rosati et al. recently developed a novel murine $T$-cell receptor which recognized human NY-ESO-1 (29). This receptor was created in part from HLA-A*201 transgenic mice harboring a human a-NE1 sequence which was then injected into a viral vector and transduced to a patient's peripheral blood lymphocytes. These "educated" lymphocytes exhibited a marked (90\%) efficiency in vitro. At present, this treatment strategy is being tested in advanced-stage melanoma patients
(NCT01350401), advanced-stage NY-ESO-1+ solid tumors (NCT01967823, NCT01697527), and in combination with other targeted therapies (NCT02070406).

\section{NY-ESO-1 expression in sarcomas}

Cronwright et al. documented normal expression of the NY-ESO-1 CTA in ex vivo cultured postnatal bone marrow and fetal liver MSCs of first-trimester fetuses (17). NYESO-1 expression is down-regulated as MSC differentiate, eventually to undetectable levels in mature mesenchymal tissues.

In their examination of tissues from 36 patients, Ayyoub et al. demonstrated NY-ESO-1 expression in gastrointestinal stromal tumor (GIST), synovial sarcoma (SS), angiosarcoma, malignant fibrous histiosarcoma, liposarcoma and chondrosarcoma; with no activity in leiomyosarcoma or osteosarcoma (30). Testing cell lines in the same study, in vitro expression of NY-ESO-1 was found in osteosarcoma and fibrosarcoma. Endo et al.'s survey of CTA expression in various sarcomas identified high levels of NY-ESO-1 [by immunohistochemistry (IHC)] in myxoid liposarcoma (85\%), SS (47\%), myxofibrosarcoma (35\%), and conventional chondrosarcoma (6-33\%) (31).

\section{Methods of detection}

Ayyoub et al. compared NY-ESO-1 monoclonal antibody (ES121) IHC to polymerase chain reaction (PCR) in their study of 36 sarcoma patients, finding excellent correlation between those these two tests amongst tumors with strong expression of the CTA, with only a few weakly positive PCR samples showing rare positivity or negative IHC results (30).

\section{NY-ESO-1 and SS}

Given the fact that the tumor-defining SSX gene-also considered a CTA by definition-is found in SS, it is unsurprising that other CTA are involved in this tumor's pathogenesis. Yet the reason for the increased frequency of expression remains unknown. Immunohistochemical detection of NY-ESO-1 in SS has ranged from $47-80 \%$ in the literature $(31,32)$. It appears that there is no predilection for CTA expression between monophasic or biphasic histology (32).

Importantly, Robbins et al. have provided compelling evidence of tumor regression in metastatic melanomas and SS, using anti-NY-ESO-1 therapy (33). After transducing 
patient T-cells with a modified T-cell receptor against NYESO-1, patients harboring NY-ESO-1-positive metastatic tumors were given these trained T-lymphocytes and the tumoral responses recorded. Four of the six patients with SS exhibited an objective tumor response with partial tumor regression-the longest documented at 18 months. More recently, D'Angelo et al. have functionally shown that circulating CD8+ T-cells developed against NY-ESO-1 are continuously maintained for months post-infusion and promote anti-tumor activity in metastatic SS (34).

\section{NY-ESO-1 and adipocytic tumors}

After gene profiling identified NY-ESO-1 as a potential analytical target in liposarcomas (35), analysis of NY-ESO-1 expression amongst liposarcomas was undertaken. The utility of NY-ESO-1 as a diagnostic marker for identification of myxoid liposarcomas was established by Hemminger et al. (36). Eighty-nine percent (89\%) of myxoid/round cell liposarcomas exhibited immunohistochemical expression of NY-ESO-1, with the majority of cases showing strong, diffuse staining (36). In a follow-up study, Hemminger and Iwenofu showed that not only was this CTA frequently expressed in myxoid/round cell liposarcomas, but indeed it was rarely expressed in other forms of liposarcoma, leading to the conclusion that NY-ESO-1 IHC is a highly sensitive and specific marker of myxoid/round cell liposarcomas (37). Endo et al. showed similar results in pre-treatment cases, but showed markedly decreased immunohistochemical expression of NY-ESO-1 in post-treatment myxoid/ round cell liposarcomas-supporting the argument that as tumor cells are forced to mature through radiation or chemotherapy, the expression of this CTA decreases (31).

From a prognostic point-of-view, univariate analysis has shown that high-level expression of NY-ESO-1 correlates with poorer prognosis. Increased immunohistochemical expression of NY-ESO-1 was significantly correlated with tumor size, the presence of tumoral necrosis, pleomorphism and an increased round-cell component, as well advanced stage at diagnosis and poor overall prognosis (38).

Finally, from a therapeutic perspective, NY-ESO-1 appears to be a strong candidate for treatment in sarcomas, in view of the strong and homogenous nature of expression. After finding expression in $100 \%$ of 25 tumors examined, researchers at the University of Washington demonstrated in vitro destruction of myxoid liposarcoma cell lines using stimulated antigen-specific CD8+ T-cells (39). Multiple studies using NY-ESO-1-specific $\mathrm{T}$ cells are pending for patients with sarcoma (NCT03462316, NCT01343043, NCT03250325, NCT03159585) or mixed population of sarcomas concurrently with LV305 or CMB305 (NCT03450122).

\section{NY-ESO-1 and other sarcomas}

NY-ESO-1 has been shown to be at least focally expressed in malignant peripheral nerve sheath tumors, uterine $(40,41)$. Expression has not been seen in non-uterine leiomyosarcomas (41). Intriguingly, though the molecular underpinnings of GISTs have recently expanded to include novel subgrouping based on cKIT, SDH and PDGFRA/ B mutations (42), it appears that there is some involvement with CTA expression as well. At least $40 \%$ of GIST were positive for at least one CTA in a study by Perez et al., with NY-ESO-1 (E978 clone) reacting in $20 \%$ of tumors tested (43). Patients expressing at least one CTA showed poorer prognosis than those which were CTA-pan-negative. Moreover, follow-up studies showed that CTA-positive tumors demonstrated a worse response to conventional tyrosine kinase inhibitors in these tumors, with a shorter recurrence-free period for the same subset (44).

\section{NY-ESO-1 expression in non-sarcomatous tumors}

The expression of NY-ESO-1 in primitive tumor cells has led to investigations in multiple tumor subtypes over the past decade, considering the immunogenic nature of this antigen. To date, NY-ESO-1 has been implicated in breast (45-47), lung (48), thyroid (49), colorectal (46), ovarian (50) and genitourinary (51) adenocarcinomas; squamous cell carcinomas of various origins $(3,48,52)$; and unusual tumoral types including hepatic carcinomas (53), ovarian and testicular primitive tumors $(9,54)$ and melanomas (55). These studies have improved the overall understanding of NY-ESO-1 pathobiology.

In melanomas, NY-ESO-1 was associated with tumor progression and with reduced tumor infiltrating lymphocytes (56). Moreover, treatment of melanomas with engineered T-cells against the NY-ESO-1 antigen showed marked reduction in tumor burden in advanced stage patients, with two of eighteen patients showing complete responses at nearly 24 months post-treatment (33). The driving force behind these reductions is the antitumoral activity brought on by both proteasome-dependent and independent mechanisms, dependent on HLA haplotype (57). 
Similar results were seen in both in vitro and in vivo models of multiple myeloma (58).

\section{NY-ESO-1 immunotherapeutic (vaccine) strategies}

\section{Simple peptide-vaccine strategies}

Both bacterial-derived peptides (from E. coli) and yeastderived peptides (from S. cerevisiae) are presently available. Interestingly, when using these proteins as adjuvants, yeast-derived proteins seem to induce NY-ESO-1 immunoreactivity superior to bacteria (59). In 2000, Jäger et al. tested the efficacy of several NY-ESO-1 peptide sequences-against known hotspots, including p157-167, p157-165 and p155-163-in eliciting CD8+ T-cell responses in both a-NE1 seropositive and seronegative patients diagnosed with varied metastatic cancers (60). The 11-mer p157-167 peptide sequence showed quicker immunogenic responses in seronegative patients, with four of seven patients exhibiting strong immunoreactivity. Repeated p157-165 9-mer peptide injections showed a similar response but over a longer period of time. Among the five seropositive patients, both the 11-mer and 9-mer peptides showed strong and quick immunoreactivity. Most interesting in this study was that five of seven vaccinated previously-seronegative patients demonstrated developed stabilization or regression of individual metastases. Three of the vaccinated previously-seropositive patients showed disease stabilization. Notably, several patients who achieved disease stabilization or regression eventually manifested disease progression. The limitations of this study protocol prevented further vaccination of the subjects, leaving the question: would these patients could have benefited from additional supplementation to booster a-NE1 activity. Miyai et al. showed a similar robust CD8+ response via T-cell receptor $\beta$ (TCR $\beta)$ rearrangements in a patient treated with the p91-110 fragment (61).

In general, however, vaccination with synthetic peptides has been lackluster in patients, secondary to tolerance or deactivation of T-cells (24). Elevated Fas and programmed death-1 expression was elucidated on CD8+ T-cells when stimulated with NY-ESO-1 p81-88 peptide, in one study (62). Most interesting in this study was finding that combining the peptides with adjuvants-CpG in the referenced study-resulted in a marked decrease of T-cell apoptosis. Thus, it seems that combining the stimulating peptides with an alternative delivery mechanism might prove fruitful in eliciting the optimal anti-tumoral response. Similar results have been seen in seropositive patients with coadministration of adjuvants, including picibanil (OK432, a TLR4 stimulant) and montanide (ISA-51) (63-67), resiquimod (68), and CpG 7909 (65).

\section{Targeting antigen—presenting cells}

Targeting dendritic cells to increase a-NE1 response has been proposed. Tsuji et al. demonstrated that creating monoclonal antibodies harboring NY-ESO-1 antigens to target specific receptors on dendritic cells was an efficacious delivery system to increase a-NE1 CD4+ and CD8+ T-cells (69). A phase I study for CDX-1401, a modified human antibody targeting dendritic cells with adherent full-length NYESO-1, demonstrated tumor regression in two patients and stabilization of disease in 13 patients for a median of 6.7 months (70). At present, there is a study investigating the use of CDX-1401 in combination therapy for prevention of recurrence in select primary gynecologic tumors in remission (NCT02166905). With respect to treatment, a Phase $1 \mathrm{~b}$ study in patients with advanced NY-ESO-1+ cancers is investigating the usage of LV305, a dendritic celltargeting viral vector which expresses the NY-ESO-1 gene, and G305, a recombinant NY-ESO-1 protein with adjuvant (NCT02387125). LV305 was initially developed in mice showing a marked anti-tumoral response against an induced CIN.23 NY-ESO-1 antigen-expressing cell line which had been injected in these mice (71). Safety in humans has since been assessed and remains a potential viable candidate for patients with refractory disease $(72,73)$.

\section{Microbe-derived recombinant vaccines}

Utilizing microorganisms to stimulate immune response has been a strategy utilized by vaccine developers for many years. One study described the use of modified Lactobacillus plantarum expressing the cell-wall tumor antigen NYESO-1 as an advantageous immunity-inducing agent, showing a marked increase in murine CD8+ T-cells after oral administration (74). Another study involved replacing genes in Salmonella enterica with the p155-165 or p157-167 fragments, generating a robust a-NE1 response in vivo (75). The generation of novel bacteria-derived adjuvants has also peaked interest recently, including the alum-polysaccharideHH2 (APH) combination derived from Escherchia coli; this vaccine strategy was shown in a mouse model of melanoma to inhibit tumor growth (76). Recombinant vaccines with 
fowlpox have also shown promise $(77,78)$.

\section{Whole-cell vaccine strategies}

In mice, $\mathrm{Xu}$ et al. induced renal cancer cells to express NYESO-1 (79). When these cells were injected into mice bearing renal cancer cell tumors without NY-ESO-1, a significant reduction in tumor size was noted, compared to controls. The interaction of dendritic cells and T-cells with the whole renal cells bearing NY-ESO-1 was noted to be markedly increased and is thought to be the driving force behind the reduction of these cells. This study strongly suggests that even in NY-ESO-1-deficient tumors, a-NE1 could be used if, if coupled to the proper antigens, to train T-cells to target tumoral epitopes with great immunogenicity.

\section{Modified vaccine strategies}

Perhaps the most interesting part of the NY-ESO-1 vaccine story has been the creative-and remarkably effectiveway in which immunogenicity has been augmented. Nanoliposomes containing the p87-111 fragment of NYESO-1 protein combined with tetanus toxoid were injected into human dendritic cells in one experiment (80). These liposomes were targeted to human dendritic cells which showed a marked immunologic response, representing the early evidence that antigens could be precisely targeted at the molecular level in these constructs. Coupling the peptides to known vaccine-derivatives has proved useful: in another study, binding of the p157-165 domain to tetanus toxin killed tumor cells which endogenously expressed NYESO-1 (81).

Other forms of nano-delivery have been attempted. Vaccination with cholesteryl pullulan complexes (CHP) containing NY-ESO-1 protein (CHP-NY-ESO-1) demonstrated no significant response compared to controls in a small sample of NY-ESO-1+ esophageal cancer patients (82). Interestingly, patients receiving higher doses of CHP-NY-ESO-1 survived longer than those receiving lower doses in a trial by Kageyama et al., despite no evidence of tumor shrinkage in these patients (83). These results suggest that CHP-NY-ESO-1 might be useful in arresting tumor growth or spread, explaining the successfulness of coadministration with other therapies (see below).

Administration of CHP-NY-ESO-1 in eight of nine patients demonstrated a heteroclitic response to NY-ESO-1 and at least one other tumor antigen, without response to the $\mathrm{CHP} /$ bacterial products used, suggesting that perhaps some degree of homology is shared between known cancer antigens in this interaction (84).

Carbon nanotubes containing NY-ESO-1 peptides utilized in mice were effective delivery systems, showing rapid internalization of the nanotube complex by dendritic cells and a resulting increase in CD8+ T-cells. Mouse survival was accordingly increased and the tumor sizes were decreased (85).

ISCOMATRIX is an immune-stimulating complex comprised of cholesterol, phospholipid and saponin (similar to chylomicrons) utilized to deliver proteins to stimulate immunogenicity (86). Priming the immune system with ISCOMATRIX-containing compounds has shown mixed reviews: one study demonstrated no significant increase in CD8+ T-cells in patients with NY-ESO-1+ tumors (87); others have shown a robust immune response $(88,89)$. The latter studies, however, did not analyze CD8+ T-cells, but rather $\mathrm{CD} 4+\mathrm{T}_{\mathrm{reg}}$-cells and-importantly-utilized fragments of immunogenic peptides in lieu of the total NY-ESO-1 protein. Coadministration of this vaccine with cyclophosphamide shows a more robust immunologic response in advanced stage melanoma patients, likely via increased NY-ESO-1-specific CD4+ $\mathrm{T}_{\text {reg }}$-cells (90).

Data from the ISCOMATRIX trials have been followed long-term, showing that the adjuvant/delivery mechanism not only dictates the degree of immunogenic response, but also the degree of immunologic memory. Nicholaou et al. showed persistent immunity in 10 of 14 vaccine recipients after a median of two years of follow-up (91). Importantly, the degree of relapse of tumor (melanoma) in this study was notably different between those receiving NY-ESO-1 peptide and those receiving ISCOMATRIX complexed NYESO-1: 5/19 vaccinated patients relapsed compared to NYESO-1 alone or placebo.

\section{Modified buman antibody strategies}

A fascinating strategy has been to modify existing human $\operatorname{IgG}$ DNA to tailor a "ready-made" antibody without requiring an antigen interaction (92). After inserting 16 NY-ESO-1 epitopes (representing over $80 \%$ of known HLA phenotypes) into the complementary determining regions (CDR) of human IgG1, authors identified a marked increase in NYESO-1 T-cell responses (93). Consistent with in vitro results, the p157-165 11-mer peptide encoded in this scheme was the most antigenic. In mice, NY-ESO-1 cells treated with this modified human IgG antibody were controlled and showed increased long-term survival of the hosts. 


\section{Use of a-NE1 vaccines as adjuvant to chemotherapeutic intervention}

Co-administration of a-NE1 has shown promise. Mice treated with either anti-CD25, anti-CTLA4 (ipilimumab) and anti-PD-1, each co-treated with a-NE1, showed a marked increase in tumor cytotoxicity compared to respective monotherapy (93). Blockade of the CTLA4 pathway was shown to significantly increase the CD8+ T-cell response in mice receiving T. cruzi-expressing NYESO-1, indicating that this cell-cycle regulator likely plays a significant role in a-NE1 efficacy (94) (although it has been described that $T$. cruzi specifically stimulated T-cell activity via CpG motifs in TLR4) (95).

In advanced stage melanoma patients, Yuan et al. showed that compared to their seronegative counterparts, patients expressing a-NE1 antibody demonstrated a greater likelihood of benefiting from anti-CTLA4 (ipilimumab) treatment (96). Post-vaccine treatment (with subsequent a-NE1 induction) with ipilimumab showed at least partial regression of tumors in four of six (66.7\%) advanced-stage melanoma patients (70).

It is known that NY-ESO-1 expression is regulated by DNA methylation, with this process silencing CTA expression in normal cells (97). The hypothesis that augmenting the methylation of DNA to increase NY-ESO-1 expression in tumor cells as a treatment strategy was tested in epithelial ovarian carcinomas by Odunsi et al. (98). Co-administering NY-ESO-1 peptide/montanide with decitabine (a hypomethylating agent) resulted in a spontaneous increase in NY-ESO-1 expression and a-NE1 immune recognition. Disease stabilization was seen in a majority of patients tested in the phase I trial, with partial regression identified in one patient.

In summary, we have discussed the current understanding and potential of utilizing the CTA, NY-ESO-1, as a therapeutic target and in some cases can serve as a diagnostic marker. As this molecule represents a super-antigen, with marked potency, and given the unique expression profile on tumors, NY-ESO-1 represents an opportunity for both cancer prevention and cancer treatment. As the results of current immunotherapeutic trials using various forms of NY-ESO-1 unravel, better understanding of the epigenetic control of this highly immunogenic CTA will continue to evolve. This will refine better combinatorial strategies going forward in the treatment of sarcomas.

\section{Acknowledgements}

None.

\section{Footnote}

Conflicts of Interest: The authors have no conflicts of interest to declare.

\section{References}

1. Apolant H, Ehrlich P. Ueber die genese des carcinoms. Tag Dtsch Path Ges 1908:1-34.

2. McKhann CF, Jagarlamoody SM. Evidence for immune reactivity against neoplasms. Transplant Rev 1971;7:55-77.

3. Chen YT, Boyer AD, Viars CS, et al. Genomic cloning and localization of CTAG, a gene encoding an autoimmunogenic cancer-testis antigen NY-ESO-1, to human chromosome Xq28. Cytogenet Cell Genet 1997;79:237-40.

4. Chen YT, Scanlan MJ, Sahin U, et al. A testicular antigen aberrantly expressed in human cancers detected by autologous antibody screening. Proc Natl Acad Sci U S A 1997;94:1914-8.

5. Gjerstorff MF, Ditzel HJ. An overview of the GAGE cancer/testis antigen family with the inclusion of newly identified members. Tissue Antigens 2008;71:187-92.

6. Van den Eynde B. A new family of genes coding for an antigen recognized by autologous cytolytic $T$ lymphocytes on a human melanoma. J Exp Med 1995;182:689-98.

7. Takahashi K, Shichijo S, Noguchi M, et al. Identification of MAGE-1 and MAGE-4 proteins in spermatogonia and primary spermatocytes of testis. Cancer Res 1995;55:3478-82.

8. Jungbluth AA, Chen YT, Stockert E, et al. Immunohistochemical analysis of NY-ESO-1 antigen expression in normal and malignant human tissues. Int $\mathrm{J}$ Cancer 2001;92:856-60.

9. Satie AP, Rajpert-De Meyts E, Spagnoli GC, et al. The Cancer-Testis Gene, NY-ESO-1, Is Expressed in Normal Fetal and Adult Testes and in Spermatocytic Seminomas and Testicular Carcinoma In Situ. Lab Invest 2002;82:775-80.

10. von Kopylow K, Kirchhoff C, Jezek D, et al. Screening for biomarkers of spermatogonia within the human testis: a whole genome approach. Hum Reprod 2010;25:1104-12.

11. Kisseleva-Romanova E, Lopreiato R, Baudin-Baillieu A, et al. Yeast homolog of a cancer-testis antigen defines a new transcription complex. EMBO J 2006;25:3576-85.

12. Dobrynin P, Matyunina E, Malov SV, et al. The novelty of human cancer/testis antigen encoding genes in evolution. Int J Genomics 2013;2013:105108. 
13. Alpen B, Gure AO, Scanlan MJ, et al. A new member of the NY-ESO-1 gene family is ubiquitously expressed in somatic tissues and evolutionarily conserved. Gene 2002;297:141-9.

14. Cho HJ, Caballero OL, Gnjatic S, et al. Physical interaction of two cancer-testis antigens, MAGE-C1 (CT7) and NY-ESO-1 (CT6). Cancer Immun 2006;6:12.

15. Cheng YH, Wong EW, Cheng CY. Cancer/testis (CT) antigens, carcinogenesis and spermatogenesis. Spermatogenesis 2011;1:209-20.

16. Bolli M, Schultz-Thater E, Zajac P, et al. NY-ESO-1/ LAGE-1 coexpression with MAGE-A cancer/testis antigens: a tissue microarray study. Int J Cancer 2005;115:960-6.

17. Cronwright G, Le Blanc K, Gotherstrom C, et al. Cancer/ testis antigen expression in human mesenchymal stem cells: down-regulation of SSX impairs cell migration and matrix metalloproteinase 2 expression. Cancer Res 2005;65:2207-15.

18. Cappell KM, Sinnott R, Taus P, et al. Multiple cancer testis antigens function to support tumor cell mitotic fidelity. Mol Cell Biol 2012;32:4131-40.

19. Whitehurst AW, Xie Y, Purinton SC, et al. Tumor antigen acrosin binding protein normalizes mitotic spindle function to promote cancer cell proliferation. Cancer Res 2010;70:7652-61.

20. Valmori D, Souleimanian NE, Hesdorffer CS, et al. Quantitative and qualitative assessment of circulating NYESO-1 specific CD4+ T cells in cancer-free individuals. Clin Immunol 2005;117:161-7.

21. Gnjatic S, Atanackovic D, Jager E, et al. Survey of naturally occurring CD4+ T cell responses against NYESO-1 in cancer patients: correlation with antibody responses. Proc Natl Acad Sci U S A 2003;100:8862-7.

22. Liu Y, Tian X, Leitner WW, et al. Polymeric structure and host Toll-like receptor 4 dictate immunogenicity of NYESO-1 antigen in vivo. J Biol Chem 2011;286:37077-84.

23. Jäger E, Chen YT, Drijfhout JW, et al. Simultaneous Humoral and Cellular Immune Response against CancerTestis Antigen NY-ESO-1: Definition of Human Histocompatibility Leukocyte Antigen (HLA)-A2-binding Peptide Epitopes. J Exp Med 1998;187:265-70.

24. Jager E, Nagata Y, Gnjatic S, et al. Monitoring CD8 T cell responses to NY-ESO-1: Correlation of humoral and cellular immune responses. Proc Natl Acad Sci U S A 2000;97:4760-5.

25. Neumann F, Wagner C, Kubuschok B, et al. Identification of an antigenic peptide derived from the cancer-testis antigen NY-ESO-1 binding to a broad range of HLA-DR subtypes. Cancer Immunol Immunother 2004;53:589-99.

26. Romero P, Dutoit V, Rubio-Godoy V, et al. CD8+ T-cell response to NY-ESO-1: relative antigenicity and in vitro immunogenicity of natural and analogue sequences. Clin Cancer Res 2001;7:766s-72s.

27. Fonteneau JF, Brilot F, Munz C, et al. The Tumor Antigen NY-ESO-1 Mediates Direct Recognition of Melanoma Cells by CD4+ T Cells after Intercellular Antigen Transfer. J Immunol 2016;196:64-71.

28. Sommermeyer D, Conrad H, Kronig H, et al. NYESO-1 antigen-reactive $\mathrm{T}$ cell receptors exhibit diverse therapeutic capability. Int J Cancer 2013;132:1360-7.

29. Rosati SF, Parkhurst MR, Hong Y, et al. A novel murine T-cell receptor targeting NY-ESO-1. J Immunother 2014;37:135-46.

30. Ayyoub M, Taub RN, Keohan ML, et al. The frequent expression of cancer/testis antigens provides opportunities for immunotherapeutic targeting of sarcoma. Cancer Immun 2004;4:7.

31. Endo M, de Graaff MA, Ingram DR, et al. NY-ESO-1 (CTAG1B) expression in mesenchymal tumors. Mod Pathol 2015;28:587-95.

32. Jungbluth AA, Antonescu CR, Busam KJ, et al. Monophasic and biphasic synovial sarcomas abundantly express cancer/testis antigen NY-ESO-1 but not MAGE-A1 or CT7. Int J Cancer 2001;94:252-6.

33. Robbins PF, Morgan RA, Feldman SA, et al. Tumor regression in patients with metastatic synovial cell sarcoma and melanoma using genetically engineered lymphocytes reactive with NY-ESO-1. J Clin Oncol 2011;29:917-24.

34. S PDA, Melchiori L, Merchant MS, et al. Antitumor Activity Associated with Prolonged Persistence of Adoptively Transferred NY-ESO-1(c259)T Cells in Synovial Sarcoma. Cancer Discov 2018;8:944-57.

35. Skubitz KM, Cheng EY, Clohisy DR, et al. Differential gene expression in liposarcoma, lipoma, and adipose tissue. Cancer Invest 2005;23:105-18.

36. Hemminger JA, Ewart Toland A, Scharschmidt TJ, et al. The cancer-testis antigen NY-ESO-1 is highly expressed in myxoid and round cell subset of liposarcomas. Mod Pathol 2013;26:282-8.

37. Hemminger JA, Iwenofu OH. NY-ESO-1 is a sensitive and specific immunohistochemical marker for myxoid and round cell liposarcomas among related mesenchymal myxoid neoplasms. Mod Pathol 2013;26:1204-10.

38. Iura K, Kohashi K, Hotokebuchi Y, et al. Cancer-testis antigens PRAME and NY-ESO-1 correlate with tumour 
grade and poor prognosis in myxoid liposarcoma. J Pathol Clin Res 2015;1:144-59.

39. Pollack SM, Jungbluth AA, Hoch BL, et al. NY-ESO-1 is a ubiquitous immunotherapeutic target antigen for patients with myxoid/round cell liposarcoma. Cancer 2012;118:4564-70.

40. Shurell E, Vergara-Lluri ME, Li Y, et al. Comprehensive adipocytic and neurogenic tissue microarray analysis of NY-ESO-1 expression - a promising immunotherapy target in malignant peripheral nerve sheath tumor and liposarcoma. Oncotarget 2016;7:72860-7.

41. Iura K, Kohashi K, Yasutake N, et al. Cancer-testis antigens are predominantly expressed in uterine leiomyosarcoma compared with non-uterine leiomyosarcoma. Oncol Lett 2018;15:441-6.

42. Smith SM, Coleman J, Bridge JA, et al. Molecular diagnostics in soft tissue sarcomas and gastrointestinal stromal tumors. J Surg Oncol 2015;111:520-31.

43. Perez D, Herrmann T, Jungbluth AA, et al. Cancer testis antigen expression in gastrointestinal stromal tumors: new markers for early recurrence. Int J Cancer 2008;123:1551-5.

44. Perez D, Hauswirth F, Jager D, et al. Protein expression of cancer testis antigens predicts tumor recurrence and treatment response to imatinib in gastrointestinal stromal tumors. Int J Cancer 2011;128:2947-52.

45. Ademuyiwa FO, Bshara W, Attwood K, et al. NY-ESO-1 cancer testis antigen demonstrates high immunogenicity in triple negative breast cancer. PLoS One 2012;7:e38783.

46. Mashino K, Sadanaga N, Tanaka F, et al. Expression of multiple cancer-testis antigen genes in gastrointestinal and breast carcinomas. Br J Cancer 2001;85:713-20.

47. Raghavendra A, Kalita-de Croft P, Vargas AC, et al. Expression of MAGE-A and NY-ESO-1 cancer/testis antigens is enriched in triple-negative invasive breast cancers. Histopathology 2018;73:68-80.

48. Gjerstorff MF, Pohl M, Olsen KE, et al. Analysis of GAGE, NY-ESO-1 and SP17 cancer/testis antigen expression in early stage non-small cell lung carcinoma. BMC Cancer 2013;13:466.

49. Gunda V, Frederick DT, Bernasconi MJ, et al. A potential role for immunotherapy in thyroid cancer by enhancing NY-ESO-1 cancer antigen expression. Thyroid 2014;24:1241-50.

50. Odunsi K, Qian F, Matsuzaki J, et al. Vaccination with an NY-ESO-1 peptide of HLA class I/II specificities induces integrated humoral and $\mathrm{T}$ cell responses in ovarian cancer. Proc Natl Acad Sci U S A 2007;104:12837-42.
51. Giesen E, Jilaveanu LB, Parisi F, et al. NY-ESO-1 as a potential immunotherapeutic target in renal cell carcinoma. Oncotarget 2014;5:5209-17.

52. Ries J, Mollaoglu N, Vairaktaris E, et al. Diagnostic and therapeutic relevance of NY-ESO-1 expression in oral squamous cell carcinoma. Anticancer Res 2009;29:5125-30.

53. Utsunomiya T, Inoue H, Tanaka F, et al. Expression of cancer-testis antigen (CTA) genes in intrahepatic cholangiocarcinoma. Ann Surg Oncol 2004;11:934-40.

54. Bode PK, Thielken A, Brandt S, et al. Cancer testis antigen expression in testicular germ cell tumorigenesis. Mod Pathol 2014;27:899-905.

55. Velazquez EF, Jungbluth AA, Yancovitz M, et al. Expression of the cancer/testis antigen NY-ESO-1 in primary and metastatic malignant melanoma (MM)-correlation with prognostic factors. Cancer Immun 2007;7:11.

56. Giavina-Bianchi M, Giavina-Bianchi P, Sotto MN, et al. Increased NY-ESO-1 expression and reduced infiltrating CD3 + T cells in cutaneous melanoma. J Immunol Res 2015;2015:761378.

57. Robson NC, McAlpine T, Knights AJ, et al. Processing and cross-presentation of individual HLA-A, -B, or -C epitopes from NY-ESO-1 or an HLA-A epitope for Melan-A differ according to the mode of antigen delivery. Blood 2010;116:218-25.

58. Schuberth PC, Jakka G, Jensen SM, et al. Effector memory and central memory NY-ESO-1-specific redirected $\mathrm{T}$ cells for treatment of multiple myeloma. Gene Ther 2013;20:386-95.

59. Wadle A, Mischo A, Strahl S, et al. NY-ESO-1 protein glycosylated by yeast induces enhanced immune responses. Yeast 2010;27:919-31.

60. Jager E, Gnjatic S, Nagata Y, et al. Induction of primary NY-ESO-1 immunity: CD8+ T lymphocyte and antibody responses in peptide-vaccinated patients with NY-ESO-1+ cancers. Proc Natl Acad Sci U S A 2000;97:12198-203.

61. Miyai M, Eikawa S, Hosoi A, et al. Detection and Tracking of NY-ESO-1-Specific CD8+ T Cells by High-Throughput T Cell Receptor beta (TCRB) Gene Rearrangements Sequencing in a Peptide-Vaccinated Patient. PLoS One 2015;10:e136086.

62. Muraoka D, Kato T, Wang L, et al. Peptide vaccine induces enhanced tumor growth associated with apoptosis induction in CD8+ T cells. J Immunol 2010;185:3768-76.

63. Wada H, Isobe M, Kakimi K, et al. Vaccination with NYESO-1 overlapping peptides mixed with Picibanil OK-432 and montanide ISA-51 in patients with cancers expressing 
the NY-ESO-1 antigen. J Immunother 2014;37:84-92.

64. Diefenbach CS, Gnjatic S, Sabbatini P, et al. Safety and immunogenicity study of NY-ESO-1b peptide and montanide ISA-51 vaccination of patients with epithelial ovarian cancer in high-risk first remission. Clin Cancer Res 2008;14:2740-8.

65. Karbach J, Gnjatic S, Bender A, et al. Tumor-reactive CD8+ T-cell responses after vaccination with NY-ESO-1 peptide, CpG 7909 and Montanide ISA-51: association with survival. Int J Cancer 2010;126:909-18.

66. Kakimi K, Isobe M, Uenaka A, et al. A phase I study of vaccination with NY-ESO-1f peptide mixed with Picibanil OK-432 and Montanide ISA-51 in patients with cancers expressing the NY-ESO-1 antigen. Int J Cancer 2011;129:2836-46.

67. Sabbatini P, Tsuji T, Ferran L, et al. Phase I trial of overlapping long peptides from a tumor self-antigen and poly-ICLC shows rapid induction of integrated immune response in ovarian cancer patients. Clin Cancer Res 2012;18:6497-508.

68. Sabado RL, Pavlick A, Gnjatic S, et al. Resiquimod as an immunologic adjuvant for NY-ESO-1 protein vaccination in patients with high-risk melanoma. Cancer Immunol Res 2015;3:278-87.

69. Tsuji T, Matsuzaki J, Kelly MP, et al. Antibody-targeted NY-ESO-1 to mannose receptor or DEC-205 in vitro elicits dual human CD8+ and CD4+ T cell responses with broad antigen specificity. J Immunol 2011;186:1218-27.

70. Dhodapkar MV, Sznol M, Zhao B, et al. Induction of antigen-specific immunity with a vaccine targeting NYESO-1 to the dendritic cell receptor DEC-205. Sci Transl Med 2014;6:232ra51.

71. Albershardt TC, Campbell DJ, Parsons AJ, et al. LV305, a dendritic cell-targeting integration-deficient ZVex(TM)based lentiviral vector encoding NY-ESO-1, induces potent anti-tumor immune response. Mol Ther Oncolytics 2016;3:16010.

72. Pollack SM, Lu H, Gnjatic S, et al. First-in-Human Treatment With a Dendritic Cell-targeting Lentiviral Vector-expressing NY-ESO-1, LV305, Induces Deep, Durable Response in Refractory Metastatic Synovial Sarcoma Patient. J Immunother 2017;40:302-6.

73. Pollack SM. The potential of the CMB305 vaccine regimen to target NY-ESO-1 and improve outcomes for synovial sarcoma and myxoid/round cell liposarcoma patients. Expert Rev Vaccines 2018;17:107-14.

74. Mobergslien A, Vasovic V, Mathiesen G, et al. Recombinant Lactobacillus plantarum induces immune responses to cancer testis antigen NY-ESO-1 and maturation of dendritic cells. Hum Vaccin Immunother 2015;11:2664-73.

75. Meng JZ, Dong YJ, Huang H, et al. Oral vaccination with attenuated Salmonella enterica strains encoding T-cell epitopes from tumor antigen NY-ESO-1 induces specific cytotoxic T-lymphocyte responses. Clin Vaccine Immunol 2010;17:889-94.

76. Li M, Shi H, Mu Y, et al. Effective inhibition of melanoma tumorigenesis and growth via a new complex vaccine based on NY-ESO-1-alum-polysaccharide-HH2. Mol Cancer 2014;13:179.

77. Jager E, Karbach J, Gnjatic S, et al. Recombinant vaccinia/ fowlpox NY-ESO-1 vaccines induce both humoral and cellular NY-ESO-1-specific immune responses in cancer patients. Proc Natl Acad Sci U S A 2006;103:14453-8.

78. Odunsi K, Matsuzaki J, Karbach J, et al. Efficacy of vaccination with recombinant vaccinia and fowlpox vectors expressing NY-ESO-1 antigen in ovarian cancer and melanoma patients. Proc Natl Acad Sci U S A 2012;109:5797-802.

79. Xu L, Zheng J, Nguyen DH, et al. Enhancing wholetumor cell vaccination by engaging innate immune system through NY-ESO-1/dendritic cell interactions. J Immunother 2013;36:412-22.

80. Cruz LJ, Rueda F, Simon L, et al. Liposomes containing NYESO1/tetanus toxoid and adjuvant peptides targeted to human dendritic cells via the Fc receptor for cancer vaccines. Nanomedicine (Lond) 2014;9:435-49.

81. Campos-Perez J, Rice J, Escors D, et al. DNA fusion vaccine designs to induce tumor-lytic CD8+ T-cell attack via the immunodominant cysteine-containing epitope of NY-ESO 1. Int J Cancer 2013;133:1400-7.

82. Aoki M, Ueda S, Nishikawa H, et al. Antibody responses against NY-ESO-1 and HER2 antigens in patients vaccinated with combinations of cholesteryl pullulan (CHP)-NY-ESO-1 and CHP-HER2 with OK-432. Vaccine 2009;27:6854-61.

83. Kageyama S, Wada H, Muro K, et al. Dose-dependent effects of NY-ESO-1 protein vaccine complexed with cholesteryl pullulan (CHP-NY-ESO-1) on immune responses and survival benefits of esophageal cancer patients. J Transl Med 2013;11:246.

84. Kawada J, Wada H, Isobe M, et al. Heteroclitic serological response in esophageal and prostate cancer patients after NY-ESO-1 protein vaccination. Int J Cancer 2012;130:584-92.

85. de Faria PC, dos Santos LI, Coelho JP, et al. Oxidized 
multiwalled carbon nanotubes as antigen delivery system to promote superior CD8(+) T cell response and protection against cancer. Nano Lett 2014;14:5458-70.

86. Barr IG, Mitchell GF. ISCOMs (immunostimulating complexes): the first decade. Immunol Cell Biol 1996;74:8-25.

87. Chen JL, Dawoodji A, Tarlton A, et al. NY-ESO-1 specific antibody and cellular responses in melanoma patients primed with NY-ESO-1 protein in ISCOMATRIX and boosted with recombinant NY-ESO-1 fowlpox virus. Int J Cancer 2015;136:E590-601.

88. Ebert LM, MacRaild SE, Zanker D, et al. A cancer vaccine induces expansion of NY-ESO-1-specific regulatory T cells in patients with advanced melanoma. PLoS One 2012;7:e48424.

89. Mischo A, Bubel N, Cebon JS, et al. Recombinant NYESO-1 protein with ISCOMATRIX adjuvant induces broad antibody responses in humans, a RAYS-based analysis. Int J Oncol 2011;39:287-94.

90. Klein O, Davis ID, McArthur GA, et al. Low-dose cyclophosphamide enhances antigen-specific CD4(+) T cell responses to NY-ESO-1/ISCOMATRIX vaccine in patients with advanced melanoma. Cancer Immunol Immunother 2015;64:507-18.

91. Nicholaou T, Chen W, Davis ID, et al. Immunoediting and persistence of antigen-specific immunity in patients who have previously been vaccinated with NY-ESO-1 protein formulated in ISCOMATRIX. Cancer Immunol Immunother 2011;60:1625-37.

Cite this article as: Smith SM, Iwenofu OH. NY-ESO-1: a promising cancer testis antigen for sarcoma immunotherapy and diagnosis. Chin Clin Oncol 2018;7(4):44. doi: 10.21037/ cco.2018.08.11
92. Pudney VA, Metheringham RL, Gunn B, et al. DNA vaccination with T-cell epitopes encoded within $\mathrm{Ab}$ molecules induces high-avidity anti-tumor CD8+ T cells. Eur J Immunol 2010;40:899-910.

93. Xue W, Metheringham RL, Brentville VA, et al. SCIB2, an antibody DNA vaccine encoding NY-ESO-1 epitopes, induces potent antitumor immunity which is further enhanced by checkpoint blockade. Oncoimmunology 2016;5:e1169353.

94. Dos Santos LI, Galvao-Filho B, de Faria PC, et al. Blockade of CTLA-4 promotes the development of effector CD8+ T lymphocytes and the therapeutic effect of vaccination with an attenuated protozoan expressing NYESO-1. Cancer Immunol Immunother 2015;64:311-23.

95. Junqueira C, Guerrero AT, Galvao-Filho B, et al. Trypanosoma cruzi adjuvants potentiate $\mathrm{T}$ cell-mediated immunity induced by a NY-ESO-1 based antitumor vaccine. PLoS One 2012;7:e36245.

96. Yuan J, Adamow M, Ginsberg BA, et al. Integrated NYESO-1 antibody and CD8+ T-cell responses correlate with clinical benefit in advanced melanoma patients treated with ipilimumab. Proc Natl Acad Sci U S A 2011;108:16723-8.

97. De Smet C, Lurquin C, Lethe B, et al. DNA methylation is the primary silencing mechanism for a set of germ lineand tumor-specific genes with a $\mathrm{CpG}$-rich promoter. Mol Cell Biol 1999;19:7327-35.

98. Odunsi K, Matsuzaki J, James SR, et al. Epigenetic potentiation of NY-ESO-1 vaccine therapy in human ovarian cancer. Cancer Immunol Res 2014;2:37-49. 